\section{FRI0724 PREDICTORS OF PRESENTEEISM AND ABSENTEEISM IN PATIENTS COMMENCING TREATMENT WITH METHOTREXATE MONOTHERAPY OR BIOLOGIC THERAPY FOR RHEUMATOID ARTHRITIS}

S. Leggett ${ }^{1}$, M. Lunt ${ }^{1}$, A. Barton ${ }^{2}$, K. Hyrich ${ }^{1}$, K. Walker-Bone ${ }^{3}$, S.M. Verstappen ${ }^{1}$ on behalf of RAMS and BRAGGSS co-investigators. ${ }^{1}$ Arthritis Research UK Centre for Epidemiology; ${ }^{2}$ Arthritis Research UK Centre for Genetics and Genomics, The University of Manchester, Manchester; ${ }^{3}$ Arthritis Research UK/MRC Centre for Musculoskeletal Health and Work, The University of Southampton, Southampton, United Kingdom

Background: The adverse effects of RA on absenteeism (i.e. days absent from work) and presenteeism (at-work productivity loss) are increasingly recognised as an important issue. Research suggests the reasons are multifactorial with many factors such as disease severity, quality of life, and depression possibly contributing to poor work outcomes in patients with RA.

Objectives: To identify predictors of presenteeism and absenteeism over one year in patients with RA commencing treatment with methotrexate (MTX) or biologics for the first time.

Methods: Patients recruited to the Rheumatoid Arthritis Methotrexate Starters study (RAMS) or the Biologics in Rheumatoid Arthritis Genetics and Genomics Study Syndicate (BRAGGSS), and in full or part-time paid employment were included in this analysis. Demographic (e.g., age), clinical (e.g., DAS28), and psychological data (e.g., Hospital Anxiety and Depression scale (HADS)) were collected at baseline. Patients were followed up at six months and one year in both cohorts. Absenteeism in the last month (days missed), and presenteeism ( 0 $=$ no interference $-10=$ complete interference), were measured at all three points using the RA specific Work Productivity Survey (WPS-RA). Patients with at least one follow-up post baseline were included in this analysis. Due to excessive zeros in the WPS-RA, a repeated measure zero inflated negative binomial regression (ZINB) was used to test the association between baseline demographic, clinical and psychological variables and presenteeism and absenteeism over one year. Results: Data was available for 191 patients in BRAGGSS and 308 in RAMS. In BRAGGSS, the mean age was 51 years (SD 9.5), median symptom duration was 8 years (IQR $5-13$ ); $78 \%$ female. In RAMS, the mean age was 52 years (SD 9.6), median symptom duration was 8.5 months (IQR 5-23); 66\% female. At baseline, $26 \%$ (BRAGGSS) and $27 \%$ (RAMS) reported $\geq 1$ days absent from work, and the median presenteeism scores were 5 (IQR 3-7) and 4 (IQR 1-7) for BRAGGSS and RAMS respectively. Presenteeism scores significantly improved over 1 year in both cohorts (BRAGGSS: median 2 [IQR 0-5], RAMS: median 2 (IQR $0-4)$ Kruskal-Wallis $p<0.001)$ ), as did absenteeism for both cohorts although non-significant. The ZINB revealed similar predictive patterns in both cohorts for presenteeism and absenteeism (Table 1). For example, for every 0.06 increase in EQ-5D (clinically important difference), the odds of being in the zero presenteeism group (i.e., no at-work productivity loss) over one year increased by a factor of 1.2 in BRAGGSS, and was similar in RAMS. In RAMS, a one unit increase in VAS fatigue decreased the odds of having no absenteeism over one year by a factor of 0.97 .

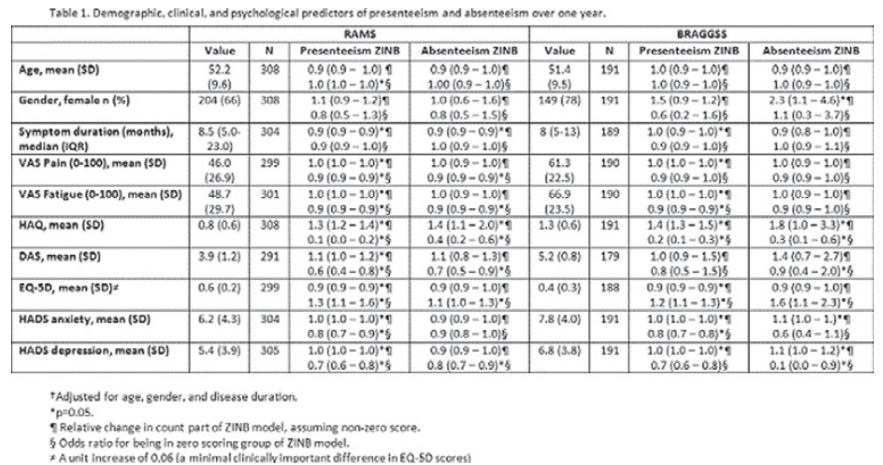

Conclusions: The results from this analysis support the notion of a multifactorial relationship between RA and work outcomes. The results suggest that greater quality of life and psychological well-being, amongst other factors, can predict improvements in presenteeism and absenteeism at one year in patients on either MTX or biologics for RA.

Acknowledgements: Funding: Pfzier I-CRP

Disclosure of Interest: None declared

DOI: 10.1136/annrheumdis-2017-eular.5077

\section{FRI0725 PREDICTION OF INTERSTITIAL LUNG DISEASE IN MIXED CONNECTIVE TISSUE DISEASE}

S. Reiseter ${ }^{1}$, R. Gunnarsson ${ }^{2}$, T.M. Aaløkken ${ }^{3}$, M.B. Lund ${ }^{4}$, J. Corander ${ }^{5}$, Ø. Molberg ${ }^{6} .{ }^{1}$ Institute of Clinical Medicine, University of Oslo; ${ }^{2}$ Dept. of Rheumatology: ${ }^{3}$ Dept. of Radiology and Nuclear Medicine; ${ }^{4}$ Dept. of Respiratory Medicine, Oslo University Hospital; ${ }^{5}$ Institute of Basic Medical Sciences; ${ }^{6}$ Institute of Clinical Medicine, University of Oslo, Oslo, Norway

Background: Mixed Connective Tissue Disease (MCTD) is characterized by high levels of autoantibodies against U1 small nuclear ribonucleoprotein (RNP) and clinical manifestations also found in Systemic Sclerosis, Systemic Lupus Erythematosus, Rheumatoid Arthritis and Polymyositis. Interstitial Lung Disease (ILD) is a severe complication in MCTD and has been reported to affect between $35 \%$ and $85 \%$ (1) in different cohorts.

Objectives: Here we present a predicting model of ILD in an unselected nationwide MCTD cohort aiming to assist clinicians in identifying MCTD patients with ILD.

Methods: 135 patients with high resolution computed tomography (CT) available for systematic evaluation were included from our nationwide MCTD cohort. Abnormal CT findings of ground glass attenuation and reticular patterns were defined as ILD. Pulmonary function tests were performed within 2 months of the HRCT examination. Serum levels of anti-RNP and Ro-52 autoantibody were determined by line immunoassay (ANA Profile 5 Euroline Blot test kit, Euroimmun, Lübeck, Germany). Logistic regression analyses were used to find the predictive factors of ILD. Variables at a significant level of $P<0.25$ where considered a candidate in the prediction model by manual backward elimination procedure in addition to age and gender.

Results: Results: 52 patients (38\%) had evidence of ILD on HRCT. The predictive model is shown in Table 1. $P=0.55$ for goodness of fit test and $P=0.80$ for area under ROC curve.

\begin{tabular}{lccccccc}
\hline & \multicolumn{3}{c}{ Univariable } & & \multicolumn{3}{c}{ Multivariable } \\
\cline { 2 - 4 } \cline { 6 - 8 } & OR & $95 \% \mathrm{Cl}$ & $\mathrm{P}$ value & & $\mathrm{OR}$ & $95 \% \mathrm{Cl}$ & $\mathrm{P}$ value \\
\hline DLCO $<60$ & 5.8 & $2.3-14.6$ & $<.001$ & & 4.6 & $1.5-13.6$ & 0.006 \\
Anti-RNP antibodies titer $>200$ & 3.0 & $1.5-7.1$ & 0.003 & & 4.9 & $1.8-13.3$ & 0.003 \\
Never arthritis & 5.4 & $2.1-13.6$ & $<.001$ & & 3.3 & $1.1-9.9$ & 0.033 \\
Age group* at HRCT & 1.0 & $1.0-1.0$ & 0.015 & & 1.6 & $1.1-2.2$ & 0.012 \\
Anti-Ro52 antibodies present & 2.6 & $1.2-5.9$ & 0.021 & & & \\
FVC $<75$ & 3.0 & $1.1-8.2$ & 0.040 & & & \\
ESR $>30$ & 2.3 & $1.0-5.0$ & 0.043 & & & \\
Sclerodactily & 2.0 & $0.94-4.0$ & 0.071 & & & \\
Male gender & 1.6 & $0.71-3.5$ & 0.268 & & & \\
\hline
\end{tabular}

${ }^{*}$ Patients were divided in 6 age groups ( $<25$ years, 26 to 35 years, 36 to 45 years, 46 to 55 years, 56-65 years and above 65 years). FVC = Forced Vital Capacity \% of predicted, DLCO = diffusing capacity of the lung for carbon monoxide $\%$ of predicted, RNP = Ribonucleoprotein, ESR = erythrocyte sedimentation rate.

Conclusions: Risk factors of ILD in MCTD are DLCO less than $60 \%$ of predicted, high titer RNP antibodies, no previous arthritis and increasing age.

References:

[1] Gunnarsson R, Hetlevik SO, Lilleby V, Molberg O. Mixed connective tissue disease. Best Pract Res Clin Rheumatol. 2016;30(1):95-111.

Disclosure of Interest: None declared

DOI: 10.1136/annrheumdis-2017-eular.1841

\section{FRI0726 LONG TERM USE OF ANALGESICS AND NSAIDS IN EARLY RA: LESSONS FROM THE CARERA STUDY}

S. Pazmino $^{1}$, R. Westhovens ${ }^{1,2}$, V. Stouten ${ }^{1}$, J. Joly ${ }^{2}$, K. Van Der Elst ${ }^{2,3}$, P. Verschueren ${ }^{1,2}$ on behalf of CareRA study group. 'Skeletal Biology and Engineering Research Centre, KU Leuven Department of Development and Regeneration; ${ }^{2}$ Rheumatology, University Hospitals Leuven; ${ }^{3}$ Skeletal Biology and Engineering Research Centre, KU Leuven Department of Public Health and Primary Care, Leuven, Belgium

Background: One might consider pain accompanying musculoskeletal conditions as a separate illness entity deserving specific treatment. A subgroup of early Rheumatoid Arthritis (RA) patients has remaining pain despite adequate disease control and this might be reflected in the use of analgesics and NSAIDs.

Objectives: To investigate the usage of analgesics and antiphlogistics prospectively in the pragmatic randomized controlled CareRA trial and describe the users of such drugs taking into account body mass index (BMI), VAS pain and DAS28CRP.

Methods: This study utilized data from the CareRA trial, a 2-year prospective investigator-initiated multicentre pragmatic RCT in patients with early RA $(\leq 1$ year) comparing different early intensive treat to target strategies, including glucocorticoid (GC) bridging, aiming for remission in all participants.

All concomitant medication for each patient was recorded, including: name, dose, frequency/timing, continuous/intermittent use, start/end date and indication (possibly/definitely RA-related or other). Meaningful sub-classifications were made (analgesics, cox2-selective and non-selective NSAIDs). We defined two subgroups with prolonged analgesic/NSAID use till w104: the pre-induction group starting before/at baseline (BL) and the post-induction group starting w28-w52, after GC step-down.

Results: From the CareRA cohort $(n=379), 90 \%$ were on analgesics/NSAIDs at some time during the study and $84 \%$ had already started before inclusion (mean 24 weeks). In general, we observed a decreasing trend in the use of analgesics/NSAIDs, with a slight increase after GC step-down. Overall, 266 patients used non-selective and 131 cox2-selective NSAIDs, 154 paracetamol and 85 opioids.

Of the total population, $15,5 \%$ (53 patients) started long-term analgesics/NSAIDs before/at BL (pre-induction group) and 13\% (45 patients) from w28-w52 (postinduction group). 
A detailed distribution of on-demand/daily analgesic/NSAID use is provided in the table below.

Table 1

\begin{tabular}{lllllc}
\hline Group of long-term users & \multicolumn{2}{c}{ NSAIDs } & Paracetamol & \begin{tabular}{c} 
Opioids \\
\cline { 2 - 5 }
\end{tabular} & $\begin{array}{c}\text { Combined } \\
\text { Non-selective Selective COX2 }\end{array}$ \\
\hline Pre-induction $(n=53)$ & $26 \%(17 \% / d)$ & $11 \%(4 \% / d)$ & $45 \%(8 \% / d)$ & $9 \%(6 \% / d)$ & $9 \%(2 \% / d)$ \\
Post-induction $(n=45)$ & $33 \%(16 \% / d)$ & $20 \%(7 \% / d)$ & $33 \%(4 \% / d)$ & $13 \%(7 \% / d)$ & $0 \%$ \\
\hline
\end{tabular}

Proportionally more patients were using NSAIDs in the post-induction group and paracetamol in the pre-induction group.

Mean DAS28CRP (BL-w104) in the pre-induction group was $3.11( \pm 0.70)$ and mean VAS pain $32.7( \pm 16.3)$. In this group $62.3 \%$ had sustained low disease activity while taking continuous analgesics/NSAIDs. Results in the post-induction group were comparable.

Analgesic/NSAID use was not significantly associated with mean (BL-w104) BMI, DAS28CRP or VAS pain.

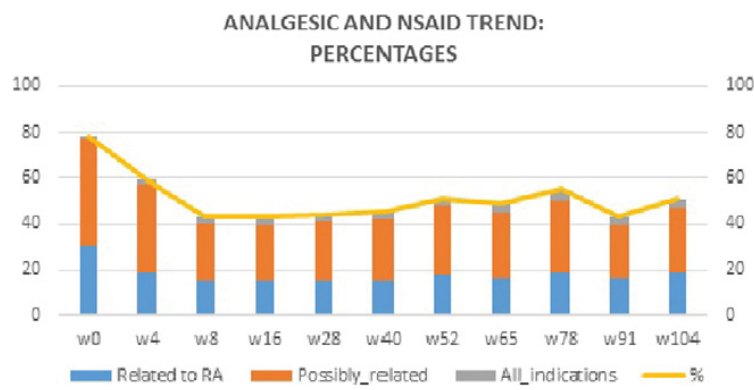

Conclusions: Analgesics/NSAID intake in early RA was high (90\%) and remarkably about $30 \%$ continued using these drugs, despite overall low disease activity. This high consumption might be explained by the ambiguous nature of arthritis-related pain and the lack of differentiation between nociceptive and non-nociceptive pain. Therefore pain management in early RA deserves more attention.

References:

[1] Verschueren, P. et al. ffectiveness of MTX with step-down glucocorticoid remission induction (COBRA Slim) vs other intensive treatment strategies for early RA in a treat-to-target approach: 1-year results of CareRA, a randomised pragmatic open-label superiority trial. Ann Rheum Dis.

Disclosure of Interest: None declared

DOI: 10.1136/annrheumdis-2017-eular.3399

\section{FRI0727 IMPACT OF MATERNAL SYSTEMIC AUTOIMMUNE RHEUMATIC DISEASES ON NEONATAL OUTCOMES: A POPULATION- LEVEL ANALYSIS}

S.O. Keeling, A. Savu, P. Kaul. Department of Medicine, University of Alberta, Edmonton, Canada

Background: The impact of systemic autoimmune rheumatic diseases (SARDS) on peripartum outcomes is not well described at a population level despite the potential for active disease in this period.

Objectives: We examined the association between SARDs and neonatal outcomes in a contemporary pregnancy cohort in the province of Alberta, Canada.

Methods: The patient population consisted of women giving birth between January 1, 2005 to December 31, 2014 ( $n=312,081)$. For women with multiple gestations during the period, one birth event was randomly selected. Women with SARDs included any of the following: systemic lupus erythematosus, systemic sclerosis, myositis and sjogren's syndrome, diagnoses based on the presence of International Classification of Disease version 9/10 codes in outpatient or inpatient records. Baseline characteristics, comorbidities, medication use (available for all births after January 1, 2009), and neonatal outcomes among women with and without SARDs were compared.

Results: Compared to women with no SARDs $(n=311,755)$, women with SARDs $(n=326,0.1 \%)$ were slightly older (SARDs 31.3 vs No SARDs 29.3 years $(p<0.01)$ ) but did not differ in terms of rural residence, ethnicity, median household income or nulliparity. However, rates of pre-term delivery, emergent cesearian section, induction, hypertensive disorders/eclampsia and mortality were higher among women with SARDs than those with no SARDs (Table 1). Offspring of women with SARDs had lower birth weights, were more likely small for gestational age (SGA), and had longer stays in neonatal ICU (Table 1). Among women with SARDs, prescription rates in the 270 days prior to delivery were highest for anti-malarials (Table 2). After multivariable adjustment, both NSAIDS use (OR (95\% CI): 5.24 $(1.57,17.52), p<0.01)$ and steroid use (OR (95\% Cl): $3.15(1.31,7.59), p<0.01)$ were significantly associated with a higher risk of preterm delivery.

Conclusions: Women with SARDs are at an increased risk of adverse outcomes during pregnancy. The association between corticosteroid and NSAID use and preterm delivery requires further investigation. Our findings suggest the need for
Table 1. Maternal and Neonatal Outcomes in SARDs vs No SARDs Mothers*

\begin{tabular}{lccc}
\hline Outcome & No SARDs \# (\%) & SARDs \# (\%) & P-value \\
\hline Pre-term Delivery & $22205(7.1)$ & $58(17.8)$ & $<0.01$ \\
C-section (Emergent) & $51132(16.4)$ & $82(25.2)$ & $<0.01$ \\
Hypertensive disorders & $20029(6.4)$ & $47(14.4)$ & $<0.01$ \\
Small for gestational age & $35047(11.2)$ & $68(20.9)$ & $<0.01$ \\
Congenital Anomaly & $5810(1.9)$ & $11(3.4)$ & 0.05 \\
Mean days in neonatal ICU & $0.8(5.0)$ & $2.3(9.6)$ & $<0.01$ \\
\hline *Sensitivity analysis utilizing primiparious SARDs vs NonSARDs women confirmed similar re-
\end{tabular}
sults.

Table 2. Unadjusted Effect of Medication Use During Pregnancy on Maternal and Neonatal Outcomes

\begin{tabular}{lccc}
\hline Outcome & Steroids & NSAIDS & Antimalarials \\
\hline \# (\%) SARDS patients on medication & $33(16.3)$ & $13(6.4)$ & $51(25.1)$ \\
SGA, OR $(95 \% \mathrm{Cl})$ & $2.06(0.91,4.67)$ & $1.08(0.29,4.12)$ & $1.54(0.74,3.20)$ \\
Gestational Diabetes, OR (95\% Cl) & $1.67(0.51,5.47)$ & $\mathrm{n} / \mathrm{a}$ & $0.91(0.28,2.93)$ \\
Preterm delivery, OR $(95 \% \mathrm{Cl})$ & $3.84(1.66,8.89)^{\star}$ & $4.96(1.55,15.85)^{\star}$ & $1.82(0.83,4.00)$ \\
C-section, OR $(95 \% \mathrm{Cl})$ & $0.88(0.41,1.90)$ & $0.98(0.31,3.11)$ & $0.57(0.29,1.13)$ \\
Hypertensive disorders of pregnancy, & & & \\
$\quad$ & $3.67(1.51,8.93)^{\star}$ & $0.50(0.06,4.03)$ & $1.23(0.51,2.99)$ \\
\hline OR $(95 \% \mathrm{Cl})$ & & &
\end{tabular}
${ }^{*} \mathrm{p}<0.05$

closer monitoring and coordinated care with obstetrics and perinatology in these high risk women.

Disclosure of Interest: None declared

DOI: 10.1136/annrheumdis-2017-eular.4561

\section{FRI0728 OSTEOARTHRITIS AND GOUT: REAL-WORLD EVIDENCE EVALUATING PATIENT CHARACTERISTICS, TREATMENT PATTERNS, AND HEALTHCARE UTILIZATION}

S. Krasnokutsky Samuels ${ }^{1}$, M. Pillinger ${ }^{1}$, S. Kabadi ${ }^{2}$, D.C. Taylor ${ }^{3}$, R. Morlock ${ }^{4}$ ${ }^{1}$ New York University, New York; ${ }^{2}$ AstraZeneca Pharmaceuticals, Gaithersburg;

${ }^{3}$ Ironwood Pharmaceuticals, Cambridge; ${ }^{4}$ YourCareChoice, Ann Arbor, United States

Background: Gout and osteoarthritis $(\mathrm{OA})$ are common in the United States, but little is known about potential associations of $O A$ and hyperuricemia/gout with clinical outcomes.

Objectives: This study examined variations in gout severity, management, and healthcare utilization among gout patients with and without $O A$.

Methods: Data were assessed from a survey of US physicians and patient chart audits. Participating physicians managed the care of $\geq 50$ patients with gout annually; chart audits were of their most recent 5 consecutive adult patients with confirmed gout. Gout severity was measured by physician global assessment, flares, organ/joint damage, and tophi. Treatment characteristics, presence of clinician-confirmed OA, and sociodemographic factors were identified. Descriptive and multivariate (stepwise logistic regression) statistics analyzed the differences among gout patients with and without clinician-confirmed comorbid OA, and assessed urate-lowering therapy (ULT) use and gout control.

Results: Overall, 1159 charts of gout patients were abstracted (230 w/ OA, $929 \mathrm{w} / 0 ; 81 \%$ male; $71 \%$ white); the proportion of patients aged $\geq 61$ was greater for those with gout and OA than those with gout but without OA $63 \%$ vs $32 \% ; P<0.001)$. Patients with gout and $O A$ had longer mean duration of gout (63 vs 41 months), were more likely to have tophi (44\% vs $19 \%$ ), joint damage (31\% vs $11 \%)$, and clinician-rated severe gout $(31 \%$ vs $12 \%)$ than those without $\mathrm{OA}$ (all $P<0.01$ ). Patients with gout and $\mathrm{OA}$ were also more likely to receive ULT $(89 \%$ vs $70 \% ; P<0.01)$, and among those receiving ULT, OA patients treated with allopurinol received a higher average daily dose (325 mg vs $296 \mathrm{mg} ; P=0.031)$. Gout patients with $O A$ were more likely to have additional comorbidities (cardiovascular disease, kidney disease, COPD, depression, diabetes, hyperlipidemia, hypertension, obesity, prostate problems [men]) and have chronic pain than those without $O A$ (all $P<0.05$ ). Gout patients with $O A$ reported more office visits (4.0 vs 3.5 ), were more likely to have an emergency department visit (17\% vs $9 \%$ ), and were more likely to require surgery for gout in the past 12 months ( $3 \%$ vs $0.3 \%$ ) (all $P<0.01$ ). In both groups, ULT use was associated with better gout control, but the specific factors predictive of ULT use and disease control varied between those with and without OA.

Conclusions: Gout patients with OA were more likely to have a greater impact on health system spending, with additional comorbidities and more severe gout than those without OA. These data suggest that gout patients with OA constitute a less healthy group in need of more careful monitoring and more aggressive gout management.

Acknowledgements: This study was sponsored by AstraZeneca.

Disclosure of Interest: S. Krasnokutsky Samuels: None declared, M. Pillinger: None declared, S. Kabadi Employee of: AstraZeneca, D. Taylor Employee of: Ironwood, R. Morlock Consultant for: AstraZeneca, Ironwood, Ardea Biosciences DOI: 10.1136/annrheumdis-2017-eular.5030 